\title{
Numerical modeling of linear photonic systems for accurate and efficient time-domain simulations
}

\author{
Yinghao Ye, ${ }^{1, *}$ Domenico Spina, ${ }^{1}$ Yufei Xing, ${ }^{2}$ Wim Bogaerts, ${ }^{2}$ and Tom Dhaene ${ }^{1}$ \\ ${ }^{1}$ IDLab, Department of Information Technology, Ghent University-imec, Ghent, Belgium \\ ${ }^{2}$ Photonics Research Group, Department of Information Technology, Ghent University-imec, Ghent, Belgium
}

compiled: May 28, 2018

\begin{abstract}
In this paper, a novel modeling and simulation method for general linear, time-invariant, passive photonic devices and circuits is proposed. This technique, starting from the scattering parameters of the photonic system under study, builds a baseband equivalent state-space model which splits the optical carrier frequency and operates at baseband, thereby significantly reducing the modeling and simulation complexity without losing accuracy. Indeed, it is possible to analytically reconstruct the port signals of the photonic system under study starting from the time-domain simulation of the corresponding baseband equivalent model. However, such equivalent models are complex-valued systems and, in this scenario, the conventional passivity constraints are not applicable anymore. Hence, the passivity constraints for scattering parameters and state-space models of baseband equivalent systems are presented, which are essential for time-domain simulations. Three suitable examples demonstrate the feasibility, accuracy and efficiency of the proposed method.
\end{abstract}

\section{Introduction}

In recent years, silicon photonic devices and circuits had a rapid development both in complexity and functionality, thanks to an increasingly mature manufacturing process. At the same time, several computer aided design (CAD) tools have emerged both in academic and industrial areas to analyze the behavior of silicon photonic designs.

Time-domain simulation of integrated photonic circuits is an essential part in the design flow, since it gives the most intuitive assessment of systems performance. For some basic photonic components, such as waveguides, time-domain simulations can be analytically addressed because of the simple underlying physical principles and equations. However, time-domain simulations cannot be performed analytically when considering more complex devices or effects caused by parasitic elements. In such scenarios, different simulation techniques, such as finite-difference time-domain (FDTD) [1], time-domain traveling wave (TDTW) [2, 3], split-step method (SSM) [4], coupled mode theory (CMT) [5], or convolution-based methods [6], operate on component or circuit level. However, a trade-off between efficiency and accuracy has to be made when using these techniques [5].

In the electronic field, a similar problem holds for distributed devices, such as nonuniform transmission lines or microstrip filters, since no compact circuit models are readily available for time-domain simulations [7]. A popular solution is based on a frequency-domain system

\footnotetext{
* Corresponding author: yinghao.ye@ugent.be
}

identification technique named vector fitting (VF) algorithm [8], which is able to build stable and passive rational models of the scattering parameters of the devices under study. Then, these frequency-domain models can be directly converted to an equivalent state-space representation in the time domain. This technique is widely applied for electronic systems, for example in [8-13].

Since the VF method is developed for linear, timeinvariant, passive systems and is based on their transfer function representation (e.g. scattering parameters), it is immediately applicable to passive photonic devices and circuits [14]. However, compared to electronic systems, the frequency range of interest for photonic systems is typically around $[187 ; 200] \mathrm{THz}$, corresponding to a wavelength of $[1.5 ; 1.6] \mu \mathrm{m}$. Such a wide range at high frequencies has a direct impact on the modeling and simulation processes, which can become very time and/or memory consuming.

To address this problem, a novel modeling method is proposed in this paper, which is based on baseband equivalent signal and system representation. In particular, the proposed modeling approach computes an accurate baseband equivalent state-space representation, starting from the scattering parameters of the photonic system under study evaluated at optical frequencies. However, such equivalent state-space model is complexvalued, and not physically realizable. Furthermore, the stability and passivity constraints on scattering parameters and state-space models of complex-valued systems, which are fundamental properties for time-domain simulations, appear yet to be missing in literature. In this paper, we rigorously discuss these conditions for the proposed baseband equivalent systems based on the classic definitions of stability and passivity to validate 
the feasibility of the proposed time-domain simulation method. The proposed technique offers two main advantages: 1) the modeling process is based on the scattering parameters, which makes it a widely applicable method for generic linear passive photonic components and circuits; 2) the state-space representation is a continuous time-domain model, which can be efficiently simulated in the time domain without involving convolution, fast Fourier transform (FFT), or inverse fast Fourier transform (IFFT), thereby making this method robust and accurate.

The paper is organized as follows. Section 2 presents an overview of the "standard" modeling approach based on the VF algorithm, while Section 3 introduces the baseband equivalent signals and systems and describes the novel proposed modeling framework. The stability and passivity constraints of such systems are discussed in Section 4. A practical guideline for the application of the proposed modeling approach is given in Section 5, while Section 6 validates the proposed method by means of three pertinent numerical examples. Conclusions are drawn in Section 7.

\section{Conventional State-Space Modeling of Photonic Systems}

In both electronics and photonics, the scattering matrix is widely used to describe the behaviors of passive devices and circuits:

$$
\boldsymbol{b}(s)=\boldsymbol{S}(s) \boldsymbol{a}(s),
$$

where $s$ is the Laplace variable, $\boldsymbol{a}(s)$ and $\boldsymbol{b}(s)$ are the forward wave and backward wave, respectively, and $\boldsymbol{S}(s)$ is the scattering matrix of the system under study, which can be obtained through simulations or measurements. The aim of the rational modeling is to find a Laplacedomain model of (1) in a pole-residue form as

$$
\boldsymbol{S}(s)=\sum_{k=1}^{K} \frac{\boldsymbol{R}_{\boldsymbol{k}}}{s-p_{k}}+\boldsymbol{D},
$$

where $\boldsymbol{D} \in \mathbb{R}^{n \times n}, \boldsymbol{R}_{k} \in \mathbb{C}^{n \times n}, k=1, \cdots, K, n$ and $K$ being the number of ports of the system under study and the number of poles used to approximate the scattering parameters, respectively. Typically, all the elements $S_{i j}(s)$ of the scattering matrix representation (2) use a common denominator polynomial and pole-set $\left[p_{1}, p_{2}, \cdots, p_{K}\right]$, where such poles are either real quantities or complex conjugate pairs [8]. The identification of poles $p_{k}$ and residue matrices $\boldsymbol{R}_{k}$ can be performed via the VF algorithm $[8,15-18]$, starting from a set of the scattering parameters under study obtained for $s_{r}=j 2 \pi f_{r}$ with $r=1, \ldots, R$.

However, it is important to note that the sign convention $e^{j w t}$ is commonly used in the electronics field to represent the incident and reflected waves in (1), while $e^{-j w t}$ is sometimes adopted in the optics field [19, 20]. Hence, the scattering matrix defined with one sign convention is the complex conjugate of the other. The VF algorithm is based on the assumption that the sign convention $e^{j w t}$ is adopted, since it has been originally developed for electromagnetic problems. In case $e^{-j w t}$ is used to define the scattering parameters under study, a simple solution is to apply the VF algorithm to the complex conjugate of the scattering matrix.

Then, the rational model (2) can be transformed to state-space form by a simple rearrangement $[17,21]$

$$
\boldsymbol{S}(s)=\boldsymbol{C}(s \boldsymbol{I}-\boldsymbol{A})^{-1} \boldsymbol{B}+\boldsymbol{D},
$$

where $\boldsymbol{A} \in \mathbb{C}^{m \times m}, \boldsymbol{B} \in \mathbb{R}^{m \times n}, \boldsymbol{C} \in \mathbb{C}^{n \times m}, \boldsymbol{D} \in \mathbb{R}^{n \times n}$, $m=n K$ and $\boldsymbol{I}$ is the identity matrix in this paper. In particular, $\boldsymbol{A}$ is a diagonal matrix with all the poles as diagonal elements while $\boldsymbol{C}$ contains all the residues, and they can be always converted to real matrices as long as the poles and residues are real or complex conjugate pairs [17].

Now, it is straightforward to convert (3) to an equivalent state-space representation in the time domain [21] as

$$
\left\{\begin{array}{cc}
\frac{\mathrm{d} \boldsymbol{x}(t)}{\mathrm{d} t}= & \boldsymbol{A x}(t)+\boldsymbol{B} \boldsymbol{a}(t) \\
\boldsymbol{b}(t) & =\boldsymbol{C x}(t)+\boldsymbol{D} \boldsymbol{a}(t)
\end{array},\right.
$$

where $\boldsymbol{x}(t) \in \mathbb{R}^{m \times 1}$ is the state vector.

Note that, fundamental properties for time-domain simulations such as the stability and passivity of models in the form (4) must be assured [13]. While the stability of VF models can be guaranteed by construction by means of suitable pole flipping schemes [8], their passivity can be checked and, eventually, enforced only after the rational model is computed by adopting passivity enforcement techniques. Indeed, due to the unavoidable numerical approximations, the rational model computed might be non-passive. Several robust passivity enforcement methods have been proposed in the literature, see for example [16-18]. Now, time-domain simulations can be carried out by solving the first-order system of ordinary differential equations (ODE) (4) via suitable numerical techniques $[22,23]$. These approaches iteratively solve (4) for a discrete set of values of the time, which are chosen via suitable algorithms (i.e. fixed or adaptive time-step). In particular, the computational cost of solving (4) depends on three main elements:

- the bandwidth of the signals considered, which define the maximum time-step $\Delta t_{\max }$ that can be adopted: $\Delta t_{\max }$ must be smaller than the highest frequency component of the signals considered;

- the numerical technique adopted to solve (4);

- the number of poles $K$ and of ports $n$ of the system under study, which directly determine the number of states $m=n K$.

The modeling technique described so far allows one to simulate any generic linear and passive system in the 
time or frequency domain and it has found extensive applications in the electronic engineering problems [8-13]. However, when it comes to photonic circuits one substantial difference arise with respect to the electronic domain: the range of frequency of interest is typically around $[187 ; 200] \mathrm{THz}$, corresponding to a wavelength of $[1.5 ; 1.6] \mu \mathrm{m}$, or even higher frequencies for shorter wavelengths. This has a major impact on the modeling and simulation complexity of the approach described so far. Indeed, a high number of poles $K$ can be required to accurately model the scattering parameters in the chosen frequency range and the passivity enforcement phase can become computationally prohibitive. Furthermore, the corresponding ODE (4) will have a high number of equations and a small time-step (of the order of femtoseconds) must be adopted to solve it.

In order to tackle these issues, a novel approach based on baseband equivalent state-space models is proposed in this contribution.

\section{Baseband Equivalent State-Space Models for Time-Domain Simulation of Photonic Systems}

The basic concepts of baseband equivalent signals and systems are first introduced in Section 3.A, given their importance in the definition of the proposed modeling approach, which is described in Section 3.B.

\section{A. Baseband Equivalent Signals and Systems}

The excitation signal of photonic systems is often an amplitude and/or phase modulated signal with optical carrier and electronic modulating signals, which can be written in the following form

$$
u(t)=A(t) \cos \left(2 \pi f_{c} t+\phi(t)\right),
$$

where $A(t)$ is the time-varying amplitude or envelope of the modulated signal, and $\phi(t)$ is the time-varying phase. In electronics or radio-frequency (RF) applications, both $A(t)$ and $\phi(t)$ relate to electronic signals, such as voltage, current or electric field. In photonics, the optical carrier frequency $f_{c}$ is much higher than the bandwidth of $A(t)$ and $\phi(t)$, given that the wavelength of light is much smaller than the one of RF signals, so the representation (5) is often called a bandpass signal.

An analytic complex-valued representation of the realvalued signal (5), called analytic signal, is introduced here as [24]

$$
u_{a}(t)=u(t)+j \mathcal{H}(u(t))=A(t) e^{j\left(2 \pi f_{c} t+\phi(t)\right)},
$$

where $\mathcal{H}(u(t))$ is the Hilbert transform of $u(t)$. In the frequency-domain equation (6) becomes

$$
U_{a}(f)=2 U(f) \operatorname{Step}(f),
$$

where $U_{a}(f)$ and $U(f)$ are the Fourier transform of $u_{a}(t)$ and $u(t)$, respectively, and $S t e p(f)$ is a unit step function
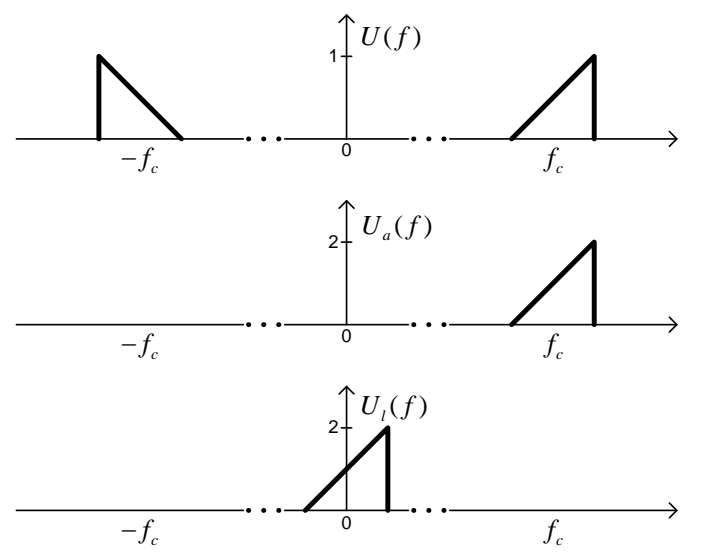

Fig. 1. Spectrum of bandpass signal $U(f)$, analytic signal $U_{a}(f)$, and baseband equivalent signal $U_{l}(f)$.

defined by

$$
\operatorname{Step}(f)=\left\{\begin{array}{c}
1, f>0 \\
\frac{1}{2}, f=0 \\
0, f<0 .
\end{array}\right.
$$

Now, the corresponding baseband equivalent signal of the bandpass signal is defined as

$$
\begin{aligned}
u_{l}(t) & =u_{a}(t) e^{-j 2 \pi f_{c}}=A(t) e^{j \phi(t)}, \\
U_{l}(f) & =2 U\left(f+f_{c}\right) \operatorname{Step}\left(f+f_{c}\right) .
\end{aligned}
$$

which can be considered as the complex envelope optical signal representation and is widely used in photonics and optical fiber communication. The relations between $u(t), \mathcal{H}(u(t))$ and $u_{l}(t)$ in the time- and frequencydomain are [24]

$$
\begin{aligned}
u(t) & =\operatorname{Re}\left(u_{l}(t) e^{j 2 \pi f_{c} t}\right), \\
\mathcal{H}(u(t)) & =\operatorname{Im}\left(u_{l}(t) e^{j 2 \pi f_{c} t}\right), \\
U(f) & =\frac{1}{2} U_{l}^{*}\left(-f-f_{c}\right)+\frac{1}{2} U_{l}\left(f-f_{c}\right),
\end{aligned}
$$

where the superscript ${ }^{*}$ denotes complex conjugate operator.

In the frequency domain, the concepts of analytic signal and baseband equivalent signal are intuitive: $U(f)$ has a symmetric spectrum with respect to the positive and negative frequencies, while $U_{a}(f)$ has only a nonzero spectrum in the positive frequencies around the carrier frequency; by shifting the spectrum of $U_{a}(f)$ in the direction of the negative frequencies of $f_{c}$ (or equivalently in the time domain by multiplying $u_{a}(t)$ with $e^{-j 2 \pi f_{c} t}$ ) leads to $U_{l}(f)$. Such relations are illustrated in Fig. 1.

If a system with impulse response $h(t)$ and frequency response $H(f)$ operates in the bandwidth $B W$ around $f c$ satisfying $f c>>B W$, then it can be considered as 


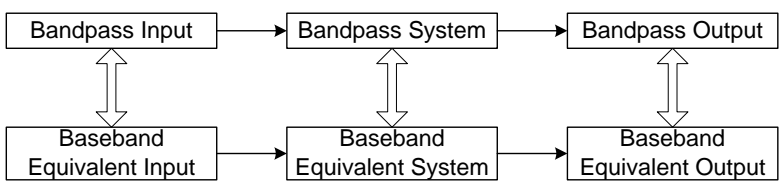

Fig. 2. Time-domain simulation of bandpass system and baseband equivalent system.

a bandpass system. Now, the corresponding baseband equivalent system can be defined by applying the same concepts described for the baseband signals. Thanks to the relations among bandpass signals and systems, and their baseband equivalents, it can be proven that the output signal of a bandpass system can be analytically recovered from the output of the corresponding baseband system, as illustrated in Fig. 2. A detailed proof is given in Appendix A.

It is important to remark that performing timedomain simulations of baseband equivalent systems allows one to efficiently recover the corresponding bandpass signals, thus avoiding expensive time-domain simulations of photonic system at optical frequencies.

\section{B. Realization of Baseband Equivalent Signals and Systems}

Baseband equivalent signals $u_{l}(t)$ can be easily computed with (9), where $u_{l}(t)$ can be a real (amplitude modulation) or a complex signal (when both amplitude and phase modulation are applied). For example, in case of a quadrature amplitude modulation (QAM), $u_{l}(t)$ can be expressed with respect to its in-phase component $I(t)=A(t) \cos \phi(t)$ and quadrature component $Q(t)=A(t) \sin \phi(t)$, as $u_{l}(t)=I(t)+j Q(t)$.

Note that, baseband equivalent signals and systems are widely used in the simulation of communication systems to simplify the modulation, demodulation and filtering process [24]. In such a scenario, continuous systems and signals are often first sampled and defined as finite discrete sequences, and then convolution, FFT, or IFFT are adopted for the time-domain simulation of the discrete-time representations of such signals and systems, which could lead to inaccurate results [6].

In this section, the goal is to build stable and passive continuous models for baseband equivalent systems in state-space form, whose time-domain simulation can also capture transient behaviors. However, a readily baseband counterpart for (4) does not exist in literature. Indeed, baseband systems have an asymmetric frequency response with respect to the positive and negative frequencies (similar to $U_{l}(f)$ in Fig. 1) resulting in a nonphysical, complex valued system, as described in details in Appendix A. The VF algorithm [8] is a technique developed for physical systems with a symmetric frequency response, which can be described with real or complex conjugate poles: this situation clearly does not hold for baseband systems and VF cannot be directly applied to the baseband response of the system under study.
In order to reach our goal, the first step is to express $\boldsymbol{a}(t), \boldsymbol{b}(t)$ and $\boldsymbol{x}(t)$ in the system of ODE (4) in the form (11), which gives

$$
\left\{\begin{array}{c}
\operatorname{Re} \frac{\mathrm{d} \boldsymbol{x}_{\boldsymbol{l}}(t) e^{j 2 \pi f_{c} t}}{\mathrm{~d} t}= \\
\boldsymbol{A} \operatorname{Re}\left(\boldsymbol{x}_{\boldsymbol{l}}(t) e^{j 2 \pi f_{c} t}\right)+\boldsymbol{B} \operatorname{Re}\left(\boldsymbol{a}_{\boldsymbol{l}}(t) e^{j 2 \pi f_{c} t}\right) \\
\operatorname{Re}\left(\boldsymbol{b}_{\boldsymbol{l}}(t) e^{j 2 \pi f_{c} t}\right)= \\
\boldsymbol{C} \operatorname{Re}\left(\boldsymbol{x}_{\boldsymbol{l}}(t) e^{j 2 \pi f_{c} t}\right)+\boldsymbol{D} \operatorname{Re}\left(\boldsymbol{a}_{\boldsymbol{l}}(t) e^{j 2 \pi f_{c} t}\right),
\end{array}\right.
$$

where $\boldsymbol{a}_{\boldsymbol{l}}(t), \boldsymbol{b}_{\boldsymbol{l}}(t)$, and $\boldsymbol{x}_{\boldsymbol{l}}(t)$ are the baseband equivalents of $\boldsymbol{a}(t), \boldsymbol{b}(t)$, and $\boldsymbol{x}(t)$, respectively. Next, by using the Hilbert transform and the relation (12) to represent $\boldsymbol{a}(t), \boldsymbol{b}(t)$ and $\boldsymbol{x}(t)$ in equation (4) leads to

$$
\left\{\begin{array}{c}
\operatorname{Im} \frac{\mathrm{d} \boldsymbol{x}_{\boldsymbol{l}}(t) e^{j 2 \pi f_{c} t}}{\mathrm{~d} t}= \\
\boldsymbol{A} \operatorname{Im}\left(\boldsymbol{x}_{\boldsymbol{l}}(t) e^{j 2 \pi f_{c} t}\right)+\boldsymbol{B} \operatorname{Im}\left(\boldsymbol{a}_{\boldsymbol{l}}(t) e^{j 2 \pi f_{c} t}\right) \\
\operatorname{Im}\left(\boldsymbol{b}_{\boldsymbol{l}}(t) e^{j 2 \pi f_{c} t}\right)= \\
\boldsymbol{C} \operatorname{Im}\left(\boldsymbol{x}_{\boldsymbol{l}}(t) e^{j 2 \pi f_{c} t}\right)+\boldsymbol{D} \operatorname{Im}\left(\boldsymbol{a}_{\boldsymbol{l}}(t) e^{j 2 \pi f_{c} t}\right) .
\end{array}\right.
$$

Equations (14) and (15) allow us to write

$$
\left\{\begin{aligned}
\frac{\mathrm{d} \boldsymbol{x}_{\boldsymbol{l}}(t) e^{j 2 \pi f_{c} t}}{\mathrm{~d} t} & =\boldsymbol{A} \boldsymbol{x}_{\boldsymbol{l}}(t) e^{j 2 \pi f_{c} t}+\boldsymbol{B} \boldsymbol{a}_{\boldsymbol{l}}(t) e^{j 2 \pi f_{c} t} \\
\boldsymbol{b}_{\boldsymbol{l}}(t) e^{j 2 \pi f_{c} t} & =\boldsymbol{C} \boldsymbol{x}_{\boldsymbol{l}}(t) e^{j 2 \pi f_{c} t}+\boldsymbol{D} \boldsymbol{a}_{\boldsymbol{l}}(t) e^{j 2 \pi f_{c} t}
\end{aligned}\right.
$$

After simple mathematical manipulations, (16) can be written as

$$
\left\{\begin{array}{rrr}
\frac{\mathrm{d} \boldsymbol{x}_{\boldsymbol{l}}(t)}{\mathrm{d} t}= & \left(\boldsymbol{A}-j 2 \pi f_{c} \boldsymbol{I}\right) \boldsymbol{x}_{\boldsymbol{l}}(t)+\boldsymbol{B} \boldsymbol{a}_{\boldsymbol{l}}(t) \\
\boldsymbol{b}_{\boldsymbol{l}}(t)= & \boldsymbol{C} \boldsymbol{x}_{\boldsymbol{l}}(t)+\boldsymbol{D} \boldsymbol{a}_{\boldsymbol{l}}(t),
\end{array}\right.
$$

which represents a realization of the baseband equivalent system by means of the state-space matrices $\left(\boldsymbol{A}-j 2 \pi f_{c} \boldsymbol{I}\right), \boldsymbol{B}, \boldsymbol{C}$ and $\boldsymbol{D}$ : in this contribution we define it as baseband state-space model. It is evident that such model can be obtained by directly shifting all the poles of the corresponding state-space model (4) of bandpass system by $j 2 \pi f_{c}$, considering that $\boldsymbol{A}$ is a diagonal complex-valued matrix with all the poles as diagonal elements, as mentioned in Section 2.

It is important to remark one difference between the representation (17) and the definition of baseband systems: in (17) the entire frequency response of the system under study is shifted in baseband, while for the baseband system introduced in Section 3.A only the frequency response at positive frequencies is shifted in baseband. However, in Appendix B it is rigorously proven that these two representation are equivalent in terms of time-domain simulations. Hence, in the rest of the contribution the expression "baseband equivalent system" does not refer to the classic definition given in Section 
3.A and Appendix A, but to the new proposed baseband equivalent "shifted" system, where the entire frequency response of the system under study is shifted in baseband.

A similar realization of baseband equivalent systems in the frequency domain, computed by shifting the poles of the transfer function of the corresponding bandpass system by $j 2 \pi f_{c}$, has been presented in the electronic domain in $[24,25]$, but the derivation is not given. Note that the time-domain simulation methods in $[24,25]$ are substantially different from the one presented here. In [24], once the transfer function of the baseband equivalent system is obtained, it is first sampled and converted to an equivalent discrete system, and then the discrete impulse response is calculated via IFFT. Finally, the time-domain behavior of the baseband equivalent system is simulated by convolution. In [25], first the inverse Laplace transform is adopted to analytically convert the baseband equivalent transfer function to a continuous impulse response, then a recursive convolution technique is used to perform time-domain simulations. In contrast, the time-domain simulation method presented in this paper directly solves the corresponding ODE, which is more straightforward. However, it is crucial to prove that fundamental properties for timedomain simulations, such as stability and passivity [13], still hold for the proposed baseband equivalent statespace representation.

\section{Passivity of Baseband Equivalent System}

The poles and residues of rational models of electronic and photonic systems are always real, or complex conjugate pairs as discussed in Section 2. However, for the baseband equivalent state-space model (17), the poles do not follow this rule anymore; furthermore the corresponding frequency response is not symmetrical with respect to positive and negative frequencies, which makes the baseband equivalent system a non-physical, complex-valued system. Finally, the most remarkable difference with respect to bandpass systems is that the impulse response of these baseband equivalent systems is not real, and with a real input, they can generate a complex output.

Then, it is important to verify if such linear, timeinvariant complex-valued systems still comply with the passivity conditions of "conventional" real-valued systems, which are listed as follows [26]:

1. Each element of $\boldsymbol{S}(s)$ is analytic in $\operatorname{Re}\{s\}>0$;

2. $\boldsymbol{I}-\boldsymbol{S}^{H}(s) \boldsymbol{S}(s)$ is a nonnegative-definite matrix for all $s$ such that $\operatorname{Re}\{s\}>0$;

3. $\boldsymbol{S}^{*}(s)=\boldsymbol{S}\left(s^{*}\right)$.

The superscript ${ }^{H}$ stands for the transpose conjugate operator. The first condition is related to causality and stability; the second one is basically a bound for $\boldsymbol{S}(s)$; the third ensures that the associated impulse response is real, which requires the system to be realvalued [27]. Evidently, the third condition is not suitable for complex-valued systems anymore. In this section, the passivity constraints for scattering parameters of baseband equivalent systems will be proposed, and a fast assessment of the passivity of the corresponding baseband equivalent state-space model will be presented.

\section{A. Passivity Constraints on Scattering Parameters of Baseband Equivalent Systems}

According to [26, 28, 29], an $n$-port electronic system is passive if, for any $\tau>-\infty$ and $\boldsymbol{v}(t) \in L_{2 n}\left(L_{2 n}\right.$ denotes the space of all vectors whose $n$ components are functions of a real variable $t$ and square integrable over $-\infty<t<\infty)$, it holds

$$
\operatorname{Re} \int_{-\infty}^{\tau} \boldsymbol{v}^{H}(t) \boldsymbol{i}(t) d t \geq 0
$$

where $\boldsymbol{v}(t), \boldsymbol{i}(t)$ are the voltage and current at the system ports. It is important to note that this definition is given not only for real signals but also for complex ones. By expressing (18) in terms of the forward $\boldsymbol{a}(t)$ and backward $\boldsymbol{b}(t)$ waves, the passivity definition becomes $[26,30,31]$

$$
\int_{-\infty}^{\tau} \boldsymbol{a}^{H}(t) \boldsymbol{a}(t)-\boldsymbol{b}^{H}(t) \boldsymbol{b}(t) d t \geq 0
$$

which is more convenient for describing photonic systems.

Following the same proof process as [26], particularly Theorem 2 and Theorem 3, the first and second passivity conditions can be derived from (19) for the complexvalued systems studied in this paper. Alternatively, the same conclusion can be obtained via the approach in Chapter II of [30] which gave simpler formal proofs using the theory of distributions. The interested reader may consult [26] and [30] for a detailed and comprehensive proof.

Therefore, in this paper we propose the following passivity constraints on the scattering parameters $\hat{\boldsymbol{S}}_{l}(s)$ of the baseband equivalent systems as:

1. $\hat{\boldsymbol{S}}_{l}(s)$ is analytic in $\operatorname{Re}(s)>0$;

2. $\boldsymbol{I}-\hat{\boldsymbol{S}}_{l}^{H}(s) \hat{\boldsymbol{S}}_{l}(s)$ is a nonnegative-definite matrix for all $s$ such that $\operatorname{Re}(s)>0$.

Note that real-valued systems need the extra condition $\boldsymbol{S}\left(s^{*}\right)=\boldsymbol{S}^{*}(s)$, which ensures that the impulse response is real, so that a real input results in a real output, and makes the system physically realizable. Furthermore, it is clearly mentioned in Section $I V$ of [26] that this requirement is independent with respect to the passivity definition in (18) and (19). Therefore this is evidently not required for the passivity of complex-valued systems which are proposed only for simulation purposes. 
4.B. Fast Passivity Assessment of Baseband Equivalent Systems

Passivity conditions require both scattering parameters $\boldsymbol{S}(s)$ and their baseband equivalent $\hat{\boldsymbol{S}}_{l}(s)$ to be bounded by unity, which implies that all singular values $\sigma$ of $\hat{\boldsymbol{S}}_{l}(s)$ are smaller than unity at all frequencies:

$$
\sigma_{i}(f)<1, \quad i=1, \ldots, n .
$$

An efficient and accurate method to assess the passivity properties of state-space models of electronic and photonic systems is based on the Hamiltonian matrix $\boldsymbol{M}[17]$ defined as

$$
\boldsymbol{M}=\left[\begin{array}{cc}
\boldsymbol{A}-\boldsymbol{B} \boldsymbol{L}^{-1} \boldsymbol{D}^{T} \boldsymbol{C} & -\boldsymbol{B} \boldsymbol{L}^{-1} \boldsymbol{B}^{T} \\
\boldsymbol{C}^{T} \boldsymbol{Q}^{-1} \boldsymbol{C} & -\boldsymbol{A}^{T}+\boldsymbol{C}^{T} \boldsymbol{D} \boldsymbol{L}^{-1} \boldsymbol{B}^{T}
\end{array}\right],
$$

where $\boldsymbol{A}, \boldsymbol{B}, \boldsymbol{C}, \boldsymbol{D}$ are the state-space matrices in (4), while $\boldsymbol{L}=\boldsymbol{D}^{T} \boldsymbol{D}-\boldsymbol{I}$ and $\boldsymbol{Q}=\boldsymbol{D} \boldsymbol{D}^{T}-\boldsymbol{I}$.

A state-space model is passive if its Hamiltonian matrix has no purely imaginary eigenvalues, since any imaginary eigenvalue indicates a crossover frequency where a singular value changes from being smaller to larger than unity, or vice versa. This approach is more reliable and efficient than sweeping the singular values over a set of discrete frequencies, especially for photonic systems which are defined over a large frequency range.

A similar Hamiltonian matrix $\hat{\boldsymbol{M}}_{l}$ for baseband equivalent systems $\hat{\boldsymbol{S}}_{l}(s)$ can be derived by following the procedure in [17], leading to

$$
\hat{\boldsymbol{M}}_{l}=\left[\begin{array}{cc}
\hat{\boldsymbol{A}}_{l}-\hat{\boldsymbol{B}}_{l} \boldsymbol{L}_{l}^{-1} \hat{\boldsymbol{D}}_{l}^{H} \hat{\boldsymbol{C}}_{l} & -\hat{\boldsymbol{B}}_{l} \boldsymbol{L}_{l}^{-1} \hat{\boldsymbol{B}}_{l}^{H} \\
\hat{\boldsymbol{C}}_{l}^{H} \boldsymbol{Q}_{l}^{-1} \hat{\boldsymbol{C}}_{l} & -\hat{\boldsymbol{A}}_{l}^{H}+\hat{\boldsymbol{C}}_{l}^{H} \hat{\boldsymbol{D}}_{l} \boldsymbol{L}_{l}^{-1} \hat{\boldsymbol{B}}_{l}^{H}
\end{array}\right],
$$

where $\hat{\boldsymbol{A}}_{l}, \hat{\boldsymbol{B}}_{l}, \hat{\boldsymbol{C}}_{l}, \hat{\boldsymbol{D}}_{l}$ are the complex-valued baseband equivalent state-space matrices, while $\hat{\boldsymbol{L}}_{l}=\hat{\boldsymbol{D}}_{l}^{H} \hat{\boldsymbol{D}}_{l}-\boldsymbol{I}$ and $\hat{\boldsymbol{Q}}_{l}=\hat{\boldsymbol{D}}_{l} \hat{\boldsymbol{D}}_{l}^{H}-\boldsymbol{I}$. The derivation of $\hat{\boldsymbol{M}}_{l}$ is shown in Appendix C.

One can observe that the only difference between $\boldsymbol{M}$ and $\hat{\boldsymbol{M}}_{l}$ is the use of the transpose conjugate operator for the state-space matrices in $\hat{\boldsymbol{M}}_{l}$, while only the transpose operator is required in $\boldsymbol{M}$. Indeed, state-space models of general electronic or photonic systems satisfy the conjugacy property $\boldsymbol{S}^{*}(s)=\boldsymbol{S}\left(s^{*}\right)$ : the corresponding scattering parameters do not change if the state-space matrices $\boldsymbol{A}, \boldsymbol{B}, \boldsymbol{C}, \boldsymbol{D}$ are replaced with their conjugate counterparts [17]. Evidently, this is not valid for the baseband equivalent systems.

Note that the eigenvalues of (22) can be obtained directly from the eigenvalues of the corresponding bandpass system (21). According to (17), by replacing $\hat{\boldsymbol{A}}_{l}$, $\hat{\boldsymbol{B}}_{l}, \hat{\boldsymbol{C}}_{l}, \hat{\boldsymbol{D}}_{l}$ in $(22)$ with

$$
\begin{aligned}
& \hat{\boldsymbol{A}}_{l}=\boldsymbol{A}-j 2 \pi f_{c} \boldsymbol{I}, \\
& \hat{\boldsymbol{B}}_{l}=\boldsymbol{B}, \\
& \hat{\boldsymbol{C}}_{l}=\boldsymbol{C}, \\
& \hat{\boldsymbol{D}}_{l}=\boldsymbol{D}
\end{aligned}
$$

gives

$$
\hat{\boldsymbol{M}}_{l}=\boldsymbol{M}-j 2 \pi f_{c} \boldsymbol{I}
$$

where $\boldsymbol{M}$ is the Hamiltonian matrix of the corresponding bandpass system. Then it is easy to derive (see Appendix C)

$$
\hat{\lambda}_{l}^{z}=\lambda^{z}-j 2 \pi f_{c}, \quad \text { for } z=1, \ldots, Z .
$$

where $Z$ is the total number of eigenvalues, while $\lambda^{z}$ and $\hat{\lambda}_{l}^{z}$ are the eigenvalues of $\boldsymbol{M}$ and $\hat{\boldsymbol{M}}_{l}$, respectively.

Hence, the following properties hold:

- If there are passivity violations in a bandpass state-space model, the corresponding baseband equivalent system (17) is not passive either.

- There is an one-to-one correspondence between the frequencies where passivity violations occurs in the state-space models of the bandpass and corresponding baseband equivalent.

- The passivity of baseband equivalent state-space models (17) can be guaranteed by applying "standard" passivity enforcement algorithm, such as $[18,32]$, to the corresponding state-space models of the bandpass systems.

\section{Proposed Modeling Framework of Photonic Sys-} tem for Time-Domain Simulations

The signals traveling through photonic systems are usually phase and/or amplitude modulated signals over a suitable optical carrier. The modulating signals are electronic ones, whose spectrum bandwidth is normally less than a few hundred gigahertz, while the carrier frequency is usually defined in the range $[187.5 ; 200] \mathrm{THz}$, corresponding to a wavelength of $[1.5 ; 1.6] \mu \mathrm{m}$.

The proposed modeling approach starts from evaluating the scattering parameters of the photonic system under study in the frequency range of interest. Next an accurate rational model is computed via the $\mathrm{VF}$ algorithm. Stability is enforced during the model-building phase via suitable pole-flipping schemes [8], while the model passivity is checked and, eventually, enforced as a postprocessing step via robust passivity enforcement methods, such as $[18,32]$. A baseband equivalent state-space representation (17) can now be obtained with guaranteed passivity by (24). Such model can be used to efficiently perform time-domain simulations. The flowchart of the proposed modeling framework is shown in Fig. 3.

In particular, when it comes to building state-space models of photonic systems for time-domain simulations, there are two options:

1. modeling the frequency range of interest, e.g. [187.5; 200] THz, noted as Model A (covering a large frequency range); 


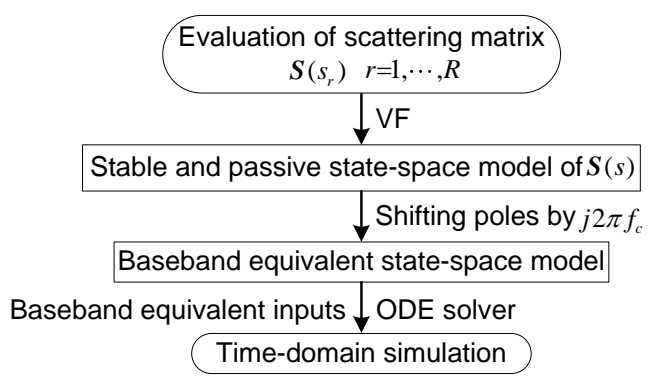

Fig. 3. Flowchart of the proposed modeling framework for time-domain simulation of photonic systems.

2. considering only the frequency range corresponding to the spectrum of the optical input signals under study around the carrier frequency, normally a few hundred gigahertz, noted as Model B (covering a small frequency range).

The corresponding baseband equivalent state-space models are indicated as Model $L A$ and Model $L B$, respectively. The modeling frequency ranges of these four models are illustrated in Fig. 4 when assuming $f_{c}=193 \mathrm{THz}$ and the spectrum of the optical input signal of interest is $300 \mathrm{GHz}$. Note that Model $A$ and $B$ can also be used directly to evaluate the behavior of the chosen photonic system in the time domain: such modeling strategies follow the approach outlined in Section 2.

Note that Model $A$ and $L A$ are likely to require more poles as compared to Model $B$ and $L B$, since they are computed over a larger bandwidth: the modeling complexity is higher and the corresponding system of ODE will be larger. If the scattering parameters under study are very dynamic in the range $[187.5 ; 200] \mathrm{THz}$, the modeling process can become prohibitively expensive, making it practically infeasible to build accurate, stable and passive models. However, this approach offers more flexibility since the corresponding models can be used

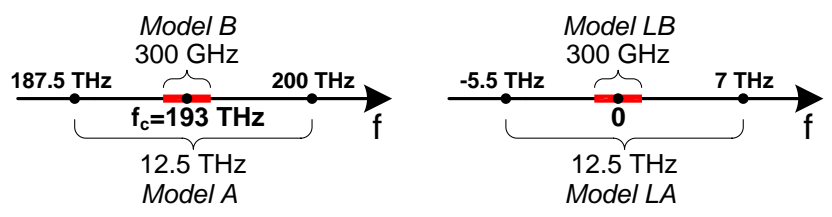

Fig. 4. Frequency ranges of $\operatorname{Model} A, L A, B$, and $L B$.

Table 1. Comparison of different modeling strategies.

\begin{tabular}{|c|c|c|c|}
\hline Models & compact & flexible & simulation at baseband \\
\hline Model $A$ & $\times$ & $\sqrt{ }$ & $\times$ \\
\hline Model $B$ & $\sqrt{ }$ & $\times$ & $\times$ \\
\hline Model $L A$ & $\times$ & $\sqrt{ }$ & $\sqrt{ }$ \\
\hline Model $L B$ & $\sqrt{ }$ & $\times$ & $\sqrt{ }$ \\
\hline
\end{tabular}

for any value of the carrier frequency in the frequency range $[187.5 ; 200] \mathrm{THz}$, while Model $B$ and $L B$ must be constructed anew for each value of the carrier frequency considered.

It is important to note that both Model $L A$ and $L B$ operate at baseband, which means that a relatively large time-step can be used to solve the corresponding ODE for time-domain simulation, thereby saving both computational time and memory storage. Table 1 compares the advantages and disadvantages of different approaches considered during the model building and time-domain simulation process.

Finally, no matter which model is used for the timedomain simulation, the modeling frequency range must be larger than or at least equal to the frequency range of the input signals considered. Indeed, no information on the scattering parameters behavior outside such modeling frequency range is provided to the $\mathrm{VF}$ algorithm: the model obtained via the $\mathrm{VF}$ approach extrapolates the scattering parameters outside the modeling frequency range. Hence, while the state-space model computed is stable and passive at $[0 ; \infty] \mathrm{Hz}$, it is not possible to guarantee its accuracy outside the modeling frequency range. Therefore, if the input signal is noisy, the spectrum of the noise should also be considered during the model building phase.

\section{Numerical Example}

This section presents three application examples of the proposed modeling and simulation technique. The scattering parameters of the photonic systems under study are evaluated via Caphe [33], while the time-domain simulations are carried out in MATLAB [34] via the routine lsim on a personal computer with Intel Core i3 processor and 8 GB RAM.

\section{A. Mach-Zehnder Interferometer}

In this example, the Mach-Zehnder interferometer (MZI) shown in Fig. 5 is studied, which is formed by two identical directional couplers (with coupling coefficient 50/50) and two waveguides with lengths $150 \mu \mathrm{m}$ (upper one) and $100 \mu \mathrm{m}$ (lower one). Both waveguides have effective index 2.35 and group index 4.3 at $1.55 \mu \mathrm{m}$, and a propagation loss of $200 \mathrm{~dB} / \mathrm{m}$. The time-domain simulation is carried out with the conventional modeling technique (in Section 2) and the proposed baseband equivalent modeling approach. For comparison, an analytic model for MZI is also built by considering the loss and dispersion of the waveguides. The directional coupler is assumed to be an ideal signal spliter or combiner, which adds a $\pi / 2$ phase delay to the cross-coupled signals. The time-domain simulation of this analyitical model is conducted as a benchmark.

The modulating signal is a smooth pulse with amplitude $1 \mathrm{~V}$, a rise/fall time of $5.7 \mathrm{ps,} \mathrm{width} \mathrm{of} 32 \mathrm{ps,} \mathrm{initial}$ delay of $18 \mathrm{ps}$, and a spectrum bandwidth of $100 \mathrm{GHz}$. An optical carrier of frequency $f_{c}=193.72 \mathrm{THz}$, which is chosen at random in the frequency range $[187.5 ; 200]$ 


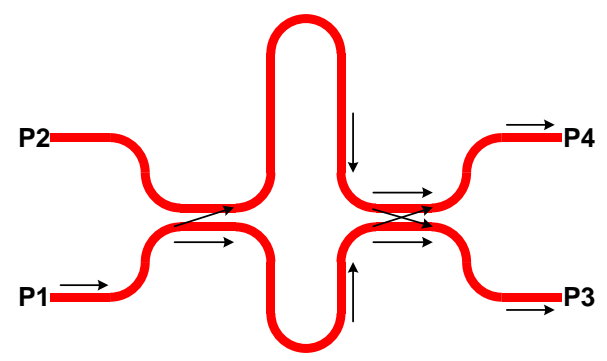

Fig. 5. Example MZI. The geometric structure of the MZI under study.

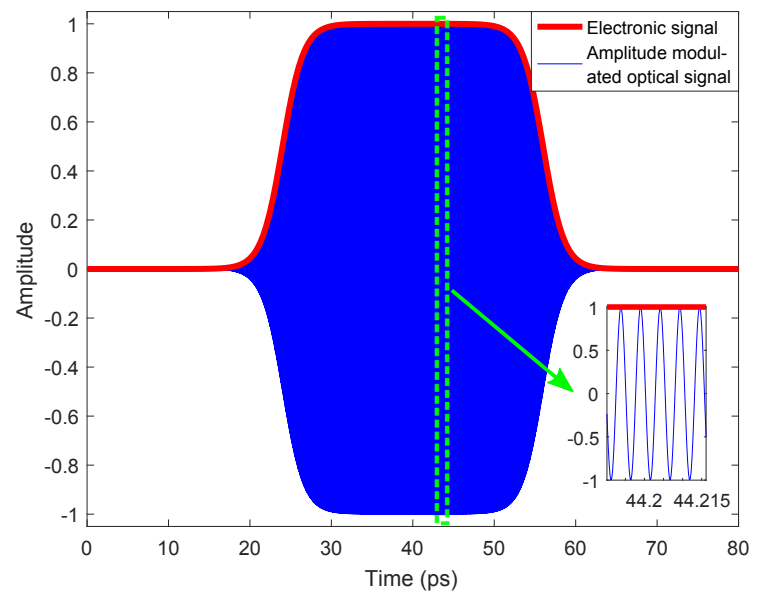

Fig. 6. Example MZI. The electronic signal and amplitude modulated optical signal for the MZI.

$\mathrm{THz}$, is used to transmit the modulated signal through the MZI. Both the modulated signal at optical frequencies and the smooth pulse are shown in Fig. 6.

Model $A$ is built starting from the MZI scattering parameters in the range $[187.5 ; 200] \mathrm{THz}$, while Model $B$ requires only the scattering parameters in $\left[f_{c}-\Delta ; f_{c}+\Delta\right]$, where the choice $\Delta=150 \mathrm{GHz}$ allows one to cover the entire spectrum of the modulated optical signal. In particular, first the frequency samples have been divided in two groups: one to compute the desired rational model (modeling data) and the other to verify its accuracy (validation data). Then Model $A$ and $B$ are built via the $\mathrm{VF}$ algorithm with 67 poles and 8 poles, respectively, aiming at a maximum absolute error of less than $-60 \mathrm{~dB}$ between the model and MZI scattering parameters. Finally, Model $L A$ and $L B$ can be derived analytically from Model $A$ and $B$, as shown in Section 3.B. The accuracy of Model $A$ and $L B$ in the frequency-domain is shown in Fig. 7 and Fig. 8, respectively, which show both the magnitude and the phase of the MZI scattering parameters obtained by Caphe and by the corresponding state-space models.

Time-domain simulations are carried out with all the four models considered; while for Model $A$ and $B$ a timestep $0.23 \mathrm{fs}$ is adopted, a time-step of $0.4 \mathrm{ps}$ can be used
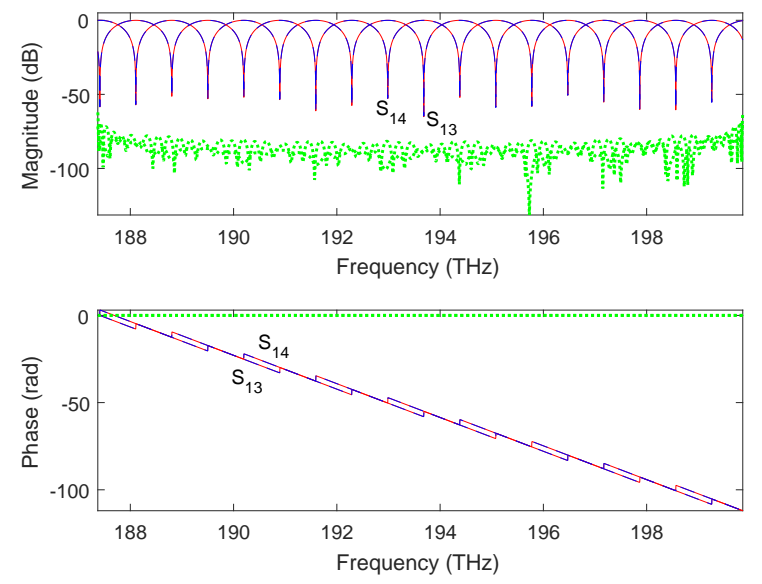

Fig. 7. Example MZI. Comparison of the magnitude (top) and phase (bottom) of the MZI scattering parameters extracted via Caphe (full blue line) and Model A (red dashed line), where the green dots represent the corresponding absolute error.
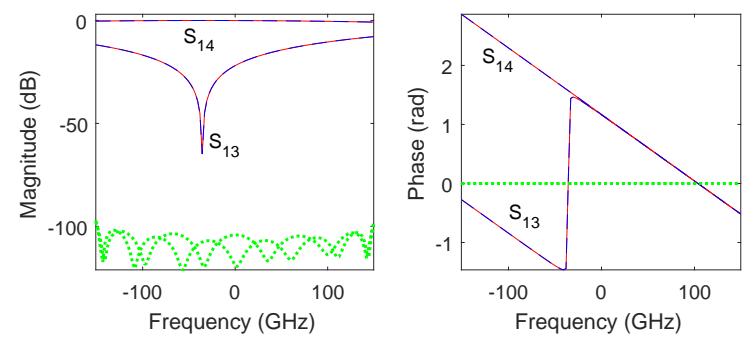

Fig. 8. Example MZI. Comparison of the magnitude (left) and phase (right) of the MZI scattering parameters extracted via Caphe (full blue line) and Model LB (red dashed line), where the green dots represent the corresponding absolute error.

for Model $L A$ and $L B$. Meanwhile, time-domain simulation of the analytic model built according the underlying physical principle of the MZI is performed in Caphe to validate the accuracy of the other models. The outputs at port P3 of Model A, Model LB, and the analytic model are shown in Fig. 9. According to Section 3, the magnitude of the outputs of Model $L B$ is the envelope of the output of Model A, and this fact is exactly illustrated by Fig. 9. In addition, it is easy to observe that the output of Model LB accurately matches the analytic model prediction.

The time for model building and time-domain simulation for all the different models are present in Table 2. It clearly shows that modeling only the small frequency range (Model $B$ and $L B$ ) rather than the large frequency range (Model $A$ and $L A$ ) consumes far less time and results in compact models. Note that the time-domain simulation at baseband with compact models, such as Model $L B$, is the most efficient, which is consistent with the analysis in Section 5 . 


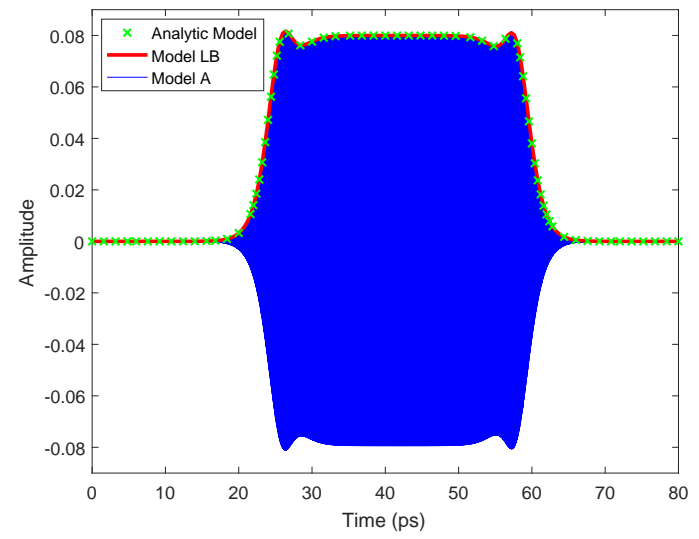

Fig. 9. Example MZI. The output at port $P 3$ of the MZI, the red line is the absolute value of the complex signal obtained by the time-domain simulation of Model $L B$, the blue line is the corresponding signal from Model $A$, while the marker $\times$ denotes the same signal from the analytic model.

Table 2. Example MZI. Efficiency comparison of the different modeling strategies

\begin{tabular}{|c|c|c|c|c|}
\hline Models & $\begin{array}{c}\text { Time } \\
\text { step }\end{array}$ & $\begin{array}{c}\text { Poles } \\
\text { number }\end{array}$ & $\begin{array}{c}\text { Model } \\
\text { building }\end{array}$ & $\begin{array}{c}\text { Time-domain } \\
\text { simulation }\end{array}$ \\
\hline Model $A$ & $0.23 \mathrm{fs}$ & 67 & $2.10 \mathrm{~s}$ & $35.66 \mathrm{~s}$ \\
\hline Model $B$ & $0.23 \mathrm{fs}$ & 8 & $0.028 \mathrm{~s}$ & $2.16 \mathrm{~s}$ \\
\hline Model $L A$ & $0.4 \mathrm{ps}$ & 67 & $2.10 \mathrm{~s}$ & $0.49 \mathrm{~s}$ \\
\hline Model $L B$ & $0.4 \mathrm{ps}$ & 8 & $0.028 \mathrm{~s}$ & $0.024 \mathrm{~s}$ \\
\hline
\end{tabular}

Finally, the following test illustrates the importance of choosing the correct modeling frequency range, as mentioned in Section 5. Let us assume an electronic pulse signal with width of $1 \mathrm{ps}$ and spectrum in the range $[0 ; 6]$ THz as the input signal of Model $L A$ and $L B$ of the MZI. The corresponding output at port $P 3$ is shown in Fig. 10: Model $L A$ still gives very accurate results compared to the analytic model, while the output of Model $L B$ is not even close to the benchmark. The reason is that the modeling frequency range $(12.5 \mathrm{THz})$ of Model LA covers the spectrum of the input signal, but this does not hold for Model LB.

\section{B. Ring Resonator}

In this example, a double ring resonator (RR) is composed of two rings and two waveguides, and designed as a narrow band flat-top filter, as shown in Fig. 11. The two rings have different circumferences $20 \mu \mathrm{m}$ (lower one) and $20.01 \mu \mathrm{m}$ (upper one), resulting in slightly different $R_{1}$ and $R_{2}$. The ring waveguides and bus waveguides have effective index 2.35 and group index 4.3 at wavelength $1.55 \mu \mathrm{m}$. The coupling coefficient between waveguides and rings is 0.2 , while the same parameter between two rings is 0.03 .

First, the Model $A$ of the ring resonator is built in the

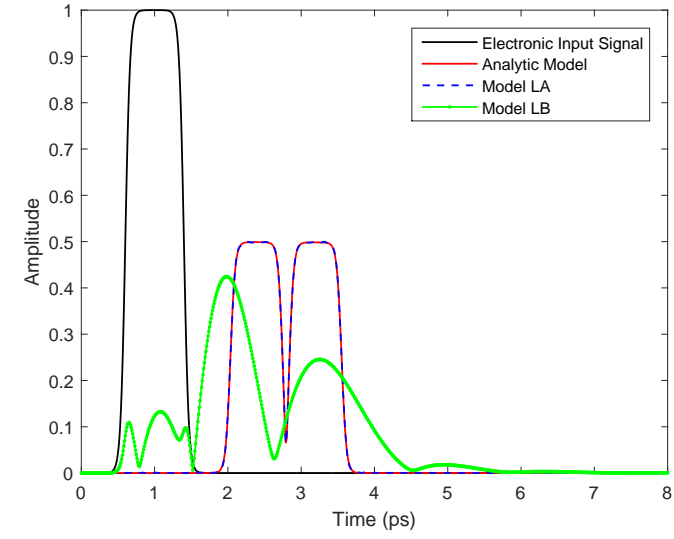

Fig. 10. Example MZI. Time-domain simulation of Model LA and $L B$ with very narrow pulse input signal. The black line is the electronic input signal, the red solid line is the output at port $P 3$ of the analytic model, while the blue dashed line and green dotted line indicate the outputs at the same port of Model $L A$ and $L B$, respectively.

range $[187.5 ; 200] \mathrm{THz}$ with 22 poles, while Model $B$ is computed with 6 poles in the range $\left[f_{c}-\Delta ; f_{c}+\Delta\right]$, with $f_{c}=195.75 \mathrm{THz}$ and $\Delta=450 \mathrm{GHz}$. The maximum absolute error of both models is less than $-65 \mathrm{~dB}$. Next, Model $L B$ is directly derived by shifting the poles of model $B$. Figures 12 and 13 describe the frequencydomain accuracy for Model $A$ and $L B$, respectively. In this example, a 4-QAM (Quadrature phase-shift keying) modulated input signal is used for time-domain simulations. The in-phase I and quadrature Q parts of the modulating signal are the 4-bits sequences $(-1,-1,1,1)$ and $(-1,1,-1,1)$, respectively, where each bit lasts for 20 ps. As shown in Fig. 14, the modulating signals are realistic analog signals, for example affected by overshoot and undershoot. As mentioned in Section 3.B, the baseband equivalent of the modulated input signal can be easily calculated, since I and Q are its real and imaginary parts, respectively.

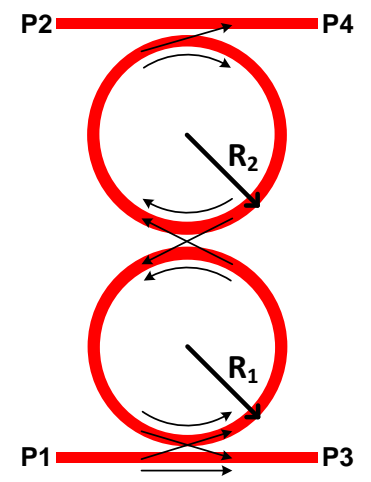

Fig. 11. Example RR. The geometric structure of the double ring resonator. 

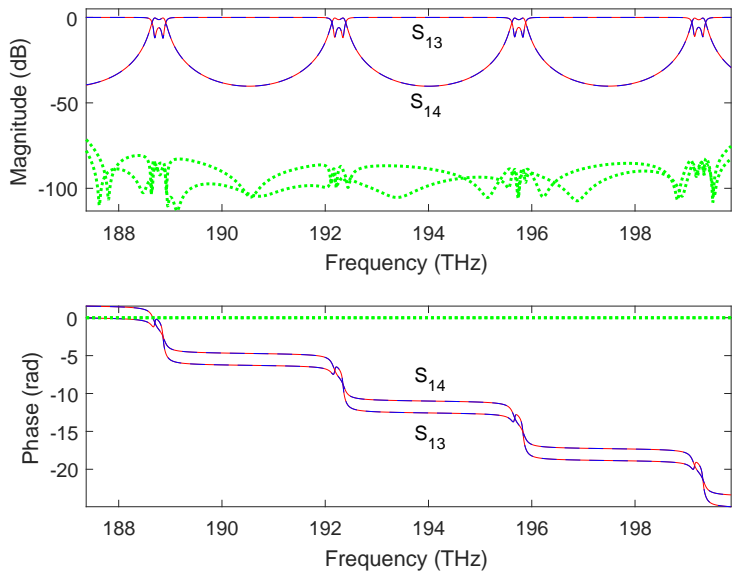

Fig. 12. Example RR. Comparison of the magnitude (top) and phase (bottom) of the ring resonator scattering parameters extracted via Caphe (full blue line) and Model A (red dashed line), where the green dots represent the corresponding absolute error.
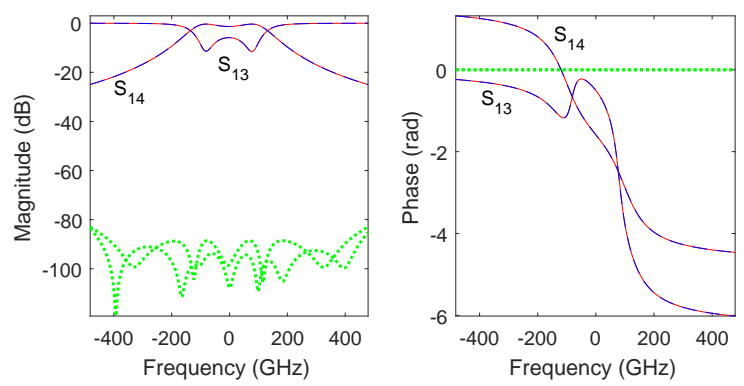

Fig. 13. Example RR. Comparison of the magnitude (left) and phase (right) of the ring resonator scattering parameters extracted via Caphe (full blue line) and Model LB (red dashed line), where the green dots represent the corresponding absolute error.

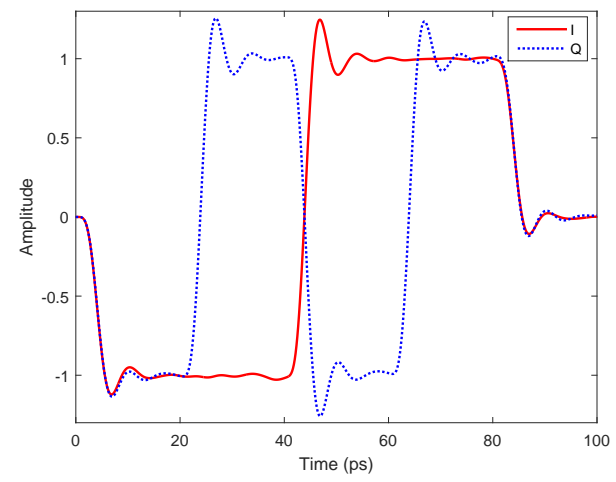

Fig. 14. Example RR. The modulating signals: in-phase part $\mathrm{I}$ and quadrature part $\mathrm{Q}$.

After conducting the proposed time-domain simula-

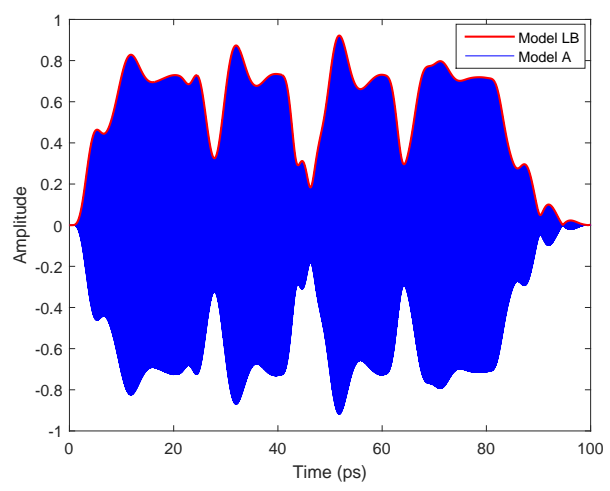

Fig. 15. Example RR. The output at port $P 3$ of the double ring resonator, the red line is the absolute value of the complex signal obtained by the time-domain simulation of Model $L B$, while the blue line is the corresponding signal from Model $A$.
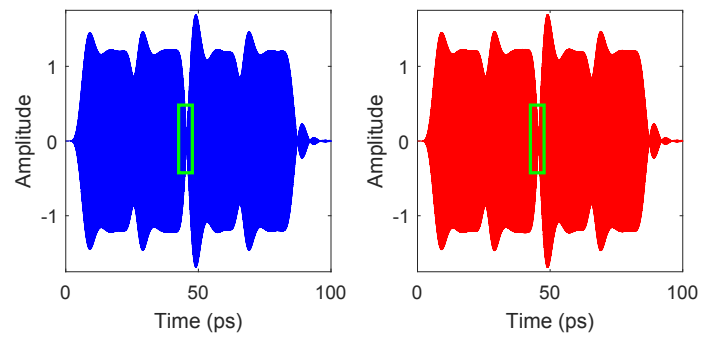

Fig. 16. Example RR. The output at port $P 4$ of the double ring resonator. Left: the output of Model A. Right: the recovered bandpass output from Model $L B$.

tion, the outputs of Model LB are complex, and their magnitude are the envelopes of the outputs of Model $A$ as shown in Fig. 15. Note that the outputs of model $A$ can be analytically recovered from the outputs of Model $L B$, according to (B3). Hence, Fig. 16 shows a side by side comparison of the output of Model $A$ at port $P 4$ and the corresponding value recovered from Model $L B$. For a better observation of the accuracy of the recovered signal, Fig. 17 shows a zoom of Fig. 16 around $t=45.6$ ps, which demonstrates an excellent agreement.

As far as the computational times are concerned, building the Model $A$ and $L B$ required $0.28 \mathrm{~s}$ and $0.04 \mathrm{~s}$ respectively while their time-domain simulations took $9.29 \mathrm{~s}$ and $0.05 \mathrm{~s}$, respectively, which clearly demonstrates the superior efficiency of the proposed technique when dealing with amplitude and phase modulated signals.

\section{C. Lattice Filter}

A fifth order filter with a Chebyshev window, designed by using a discrete finite impulse response (FIR) filter design method [35], is realized via a Mach-Zehnder interferometer lattice filter (LF) [36]. As illustrated in Fig. 18, it is formed by six directional couplers with 


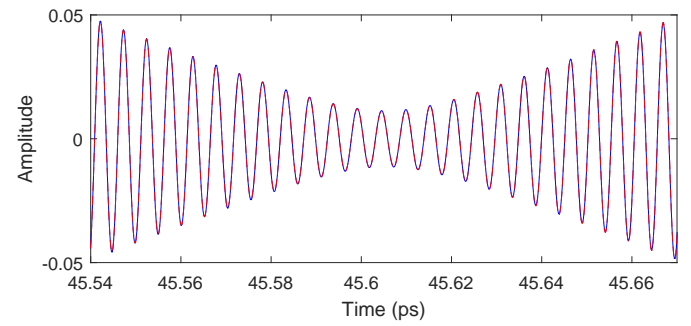

Fig. 17. Example RR. A zoom of the output at port P4 of the double ring resonator around $t=45.6 \mathrm{ps}$ (the green rectangular area in Fig. 16). The blue line is used for Model $A$, while the red dash line is the recovered bandpass output from Model $L B$.

power coupling coefficients of $0.008,0.067,0.175,0.175$, $0.067,0.008$, and waveguides with a length difference of $179 \mu \mathrm{m}$ between the upper and lower ones, whose effective and group index are 2.30 and 4.18 , respectively. In practice, due to process variations, when manufacturing photonic devices geometrical or optical parameters can vary in a relatively small range around their nominal value [37]; which in turn can lead to variations in the device frequency response, such as frequency shifts. In this example, we study the time-domain influence of frequency shifts in the response of the lattice filter via an eye diagram analysis.

For eye diagram analysis, the input signal and timedomain simulation should last a relatively long period of time (long bits sequence), which could make the timedomain simulation of Model $A$ and $B$ unfeasible. In this example, a pseudo-random sequence of 1000 bits, with a bit time of 30 ps and a Gaussian jitter having a standard deviation of $1.5 \mathrm{ps}$ is used as modulating signal $A(t)$. The amplitude of such signal up to $1 \mathrm{~ns}$ is shown in Fig. 19 . The total number of time-steps required for time-domain simulations of Model $A$ and $B$ with such input signal is 60 million ( $30 \mathrm{~ns} / 0.5 \mathrm{fs}$ ), while this number reduces to only 30,000 time-steps $(30 \mathrm{~ns} / 1 \mathrm{ps})$ for Model $L A$ and $L B$.

The scattering matrices of the lattice filter are computed in the range $[187.5 ; 200] \mathrm{THz}$. However, due to the dynamic behavior of the filter frequency response in such a wide bandwidth, the modeling complexity of

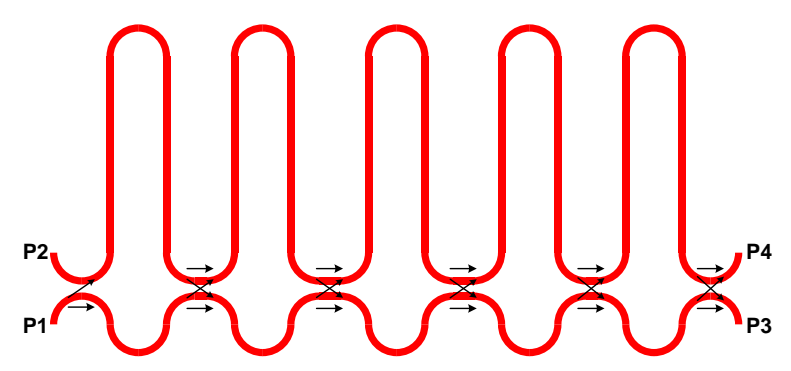

Fig. 18. Example LF. The geometric structure of the MachZehnder interferometer lattice filter.

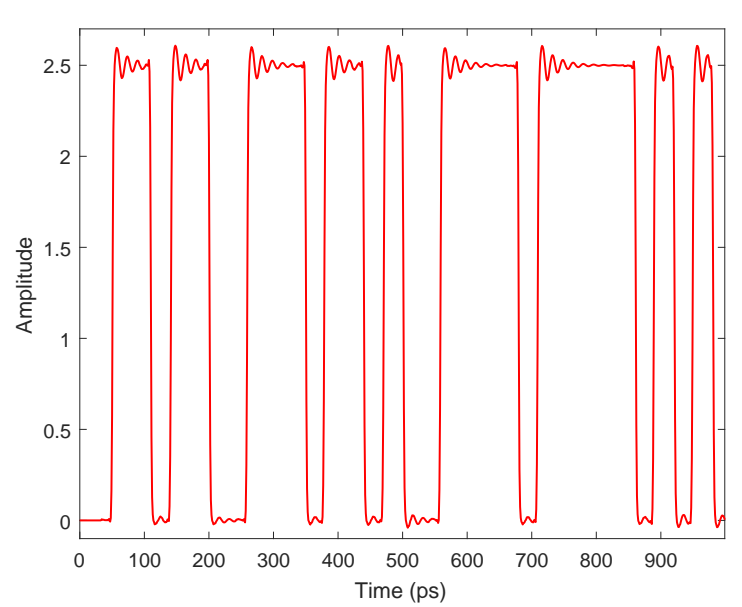

Fig. 19. Example LF. Pseudo-random sequence of 1000 bits for $t \in[0 ; 1] \mathrm{ns}$.

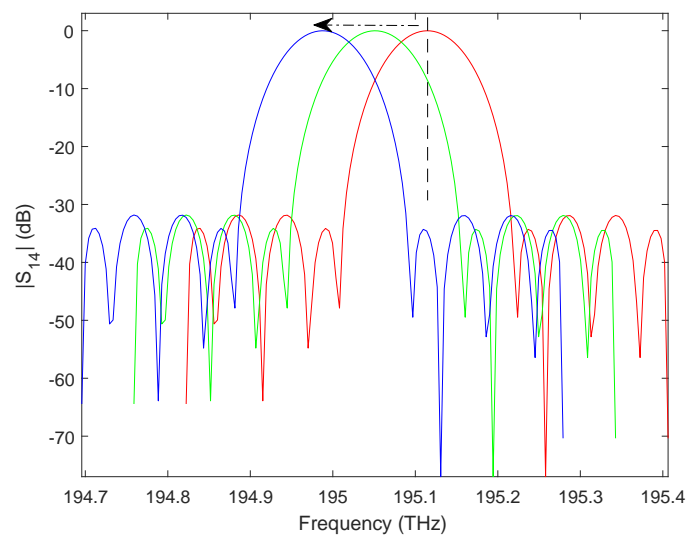

Fig. 20. Example LF. Shift of the center frequency of passband of the lattice filter due to the tolerances of the manufacturing process.

Model $A(L A)$ is very high. Considering that the efficiency and accuracy of Model $L B$ have been already demonstrated in Sections 6.A and 6.B, only the timedomain simulation of Model $L B$ is performed.

The sequence signal is modulated on $f_{c}=195.11$ $\mathrm{THz}(\lambda=1.5365 \mu \mathrm{m})$, which is chosen as the filter passband center frequency during the design phase. Due to manufacturing tolerances, let us assume that the center frequency can shifts to $195.05 \mathrm{THz}(\lambda=1.5370 \mu \mathrm{m})$ or $194.98 \mathrm{THz}(\lambda=1.5375 \mu \mathrm{m})$, as shown in Fig. 20. Model $L B$ is built for each one of these three situations, by adopting a pole shift of $f_{c}=195.11 \mathrm{THz}$, since the excitation signal is modulated on this frequency. In particular, the models for the three wavelength considered are built with 36 poles achieving a maximum absolute error of $-60 \mathrm{~dB}$.

Then, the time-domain simulations can be easily carried out at baseband with the pseudo-random sequence 

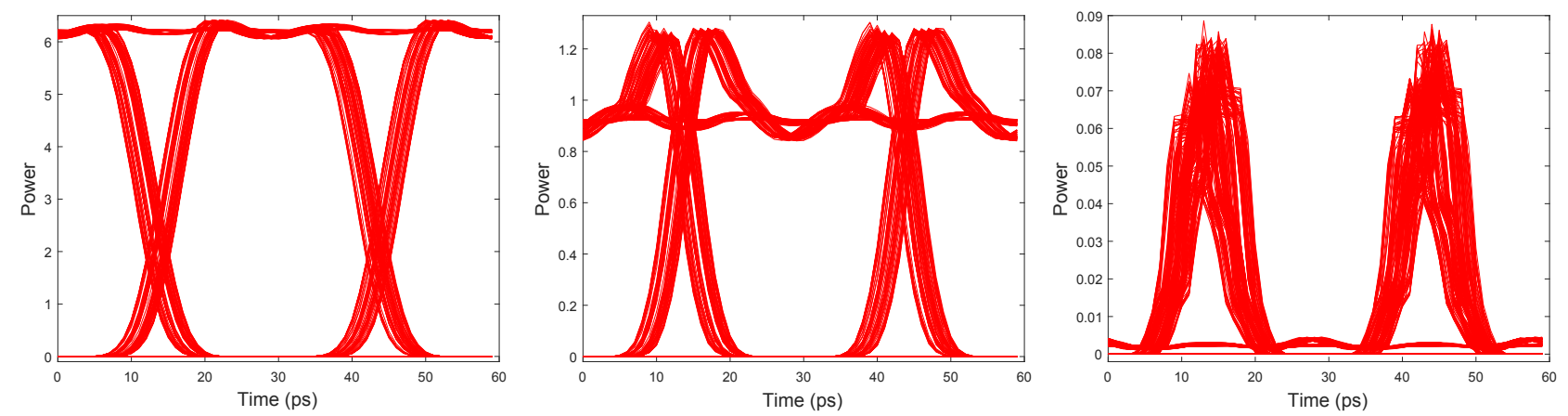

Fig. 21. Example LF. The eye diagrams at port $P 4$ of the baseband equivalent systems of the lattice filter with passband center frequency $195.11 \mathrm{THz}, 195.05 \mathrm{THz}, 194.98 \mathrm{THz}$ (from left to right).

of 1000 bits. Figure 21 shows the eye diagram of the power of the complex output signals at port $P 4$ of the three baseband equivalent systems, over a two-bit span resulting from the entire 1000-bit input stream. It is evident that the signal is completely distorted when the center frequency shift from $195.11 \mathrm{THz}$ to $194.98 \mathrm{THz}$. The computational time of the time-domain simulation for generating each eye diagram is $1.09 \mathrm{~s}$ while building each model took $1.67 \mathrm{~s}$, which is very efficient. This example shows that expensive time-domain simulations can be efficiently performed via the proposed technique, without a loss in accuracy.

\section{Conclusion}

A novel modeling and simulation technique for passive photonic components and circuits has been proposed in this paper, which is flexible, efficient, accurate and robust. Photonics systems can be characterized by the proposed baseband equivalent state-space models via the robust VF algorithm, which allows for the time-domain simulations to be conducted at baseband rather than at the optical carrier frequency. The outputs of photonic systems can be immediately recovered from the outputs of the corresponding baseband equivalent models, thereby significantly decreasing the simulation time and memory usage. The passivity conditions of the proposed baseband equivalent systems are rigorously discussed and a fast passivity assessment method for the corresponding state-space models is presented in this paper. The accuracy and efficiency of the proposed approach are verified by three suitable numerical examples.

\section{Funding}

Part of this work is supported by Research FoundationFlanders (FWO-Vlaanderen) with Grant number G013815N, and the agency Flanders Innovation \& Entrepreneurship (VLAIO) and Luceda Photonics through the MEPIC project.

\section{Appendix A Time-Domain Simulation of Baseband Equivalent Signals and Systems}

If a system with impulse response $h(t)$ and frequency response $H(f)$ operates in the bandwidth $B W$ around $f_{c}$ satisfying $f_{c}>B W$, then it can be considered as a bandpass system. Now, in a similar manner as with the baseband equivalent signal, a baseband equivalent system with impulse response $h_{l}(t)$ and frequency response $H_{l}(f)$ can be defined as [24]

$$
\begin{aligned}
h_{l}(t) & =\frac{1}{2} h_{a}(t) e^{-j 2 \pi f_{c}}, \\
H_{l}(f) & =H\left(f+f_{c}\right) \operatorname{Step}\left(f+f_{c}\right),
\end{aligned}
$$

where $h_{a}(t)$ is the analytic signal of $h(t)$ and is defined in the same way as (6).

Compared with the definition of baseband equivalent signals, a factor $1 / 2$ is introduced into the definition of baseband equivalent systems [24]. Again, the relations between $h(t), \mathcal{H}(h(t))$ and $h_{l}(t)$ in the time- and frequency-domain are

$$
\begin{aligned}
h(t) & =2 \operatorname{Re}\left(h_{l}(t) e^{j 2 \pi f_{c} t}\right), \\
\mathcal{H}(h(t)) & =2 \operatorname{Im}\left(h_{l}(t) e^{j 2 \pi f_{c} t}\right), \\
H(f) & =H_{l}^{*}\left(-f-f_{c}\right)+H_{l}\left(f-f_{c}\right) .
\end{aligned}
$$

It is important to note that baseband equivalent signals and systems are not physical, but constitute a mathematical representation developed only for simplifying analysis and simulation of bandpass signals and systems, as discussed in the following.

Let us assume that the bandpass input signal, system, and output are $u(t), h(t)$ and $r(t)$, respectively, while their corresponding Fourier transforms are indicated as $U(f), H(f)$ and $R(f)$. Then, the following relations hold

$$
\begin{aligned}
r(t) & =h(t) \otimes u(t), \\
R(f) & =H(f) U(f),
\end{aligned}
$$

where $\otimes$ represents the convolution operator. Now, the corresponding baseband equivalents of the input signal and system are $u_{l}(t), h_{l}(t), U_{l}(f), H_{l}(f)$. Hence, the output signal of the baseband equivalent system can be defined as

$$
\begin{aligned}
r_{l}(t) & =h_{l}(t) \otimes u_{l}(t), \\
R_{l}(f) & =H_{l}(f) U_{l}(f) .
\end{aligned}
$$


In the following, it is proven that the output of the baseband equivalent system $r_{l}(t), R_{l}(f)$ and the output of the bandpass system $r(t), R(f)$ have the same relations as baseband equivalent and bandpass signals (see (11) and (13)). Indeed, starting from (A6) and (A7), the following relations can be derived [24]:

$$
\begin{aligned}
R(f)=\mathscr{F}(r(t))=H(f) U(f) \\
=\frac{1}{2}\left[H_{l}\left(f-f_{c}\right)+H_{l}^{*}\left(-f-f_{c}\right)\right]\left[U_{l}\left(f-f_{c}\right)\right. \\
\left.\quad+U_{l}^{*}\left(-f-f_{c}\right)\right] \\
=\frac{1}{2}\left[H_{l}\left(f-f_{c}\right) U_{l}\left(f-f_{c}\right)+H_{l}^{*}\left(-f-f_{c}\right) U_{l}^{*}\left(-f-f_{c}\right)\right] \\
=\frac{1}{2}\left[R_{l}\left(f-f_{c}\right)+R_{l}^{*}\left(-f-f_{c}\right)\right] \\
=\frac{1}{2} \mathscr{F}\left(r_{l}(t) e^{j 2 \pi f_{c} t}+r_{l}^{*}(t) e^{-j 2 \pi f_{c} t}\right) \\
=\mathscr{F}\left(\operatorname{Re}\left(r_{l}(t) e^{j 2 \pi f_{c} t}\right)\right),
\end{aligned}
$$

where the symbol $\mathscr{F}$ represents the Fourier transform operator. Equation (A8) clearly demonstrates that $r_{l}(t)$ is the complex envelope of the bandpass system output: $r(t)$ can be immediately obtained from $r_{l}(t)$ [24].

\section{Appendix B Baseband Equivalent "Shifted" System}

In the following, we prove that the baseband equivalent "shifted" system represented by (17) is equivalent to the based equivalent system $h_{l}(t)$ in (A1), in the sense of time-domain simulations.

According to Section 3.B, the transfer function $\hat{H}_{l}(f)$ and impulse response $\hat{h}_{l}(t)$ of the proposed baseband equivalent state-space model (17) can be described as

$$
\begin{aligned}
\hat{H}_{l}(f) & =H\left(f+f_{c}\right)=H_{l}\left(f+2 f_{c}\right)+H_{l}(f), \\
\hat{h}_{l}(t) & =h(t) e^{-j 2 \pi f_{c} t},
\end{aligned}
$$

since it is obtained by shifting all the poles of the corresponding state-space model of bandpass system by $j 2 \pi f_{c}$, considering that $\boldsymbol{A}$ is a diagonal complex-valued matrix with all the poles as diagonal elements.

By comparing the results obtained in (B1) and (B2) to the baseband equivalent system definition given in (A1) and (A2), one difference is clear: only the frequency response of $H(f)$ at positive frequencies is shifted by $f_{c}$ in the definitions (A1) and (A2), while in (B1) and (B2) the entire frequency response of the bandpass system considered is shifted. This difference is illustrated in Fig. 22.

Then it is proven that the relation (A8) still holds for baseband equivalent "shifted" systems calculated by means of (B1) and (B2). Indeed, the output signals of the bandpass system in the frequency-domain can be written as
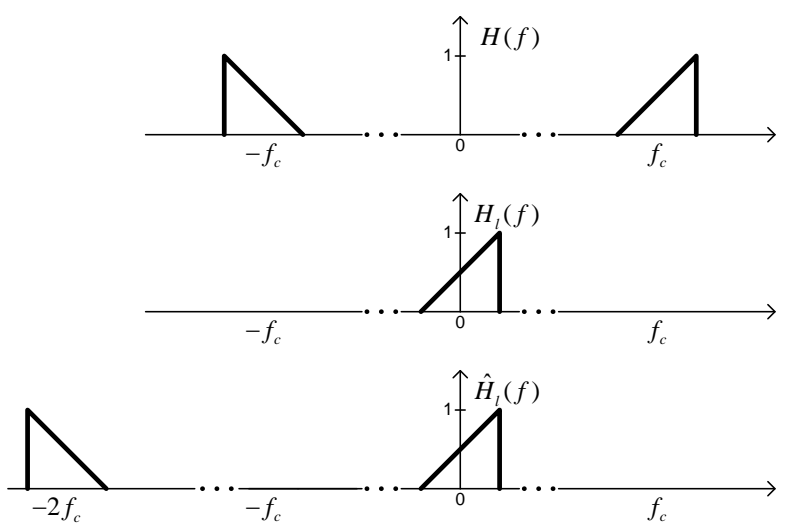

Fig. 22. Spectrum of bandpass system $H(f)$, baseband equivalent system $H_{l}(f)$, and baseband equivalent "shifted" system $\hat{H}_{l}(f)$.

$$
\begin{aligned}
& R(f)=\mathscr{F}(r(t))=H(f) U(f) \\
& =\frac{1}{2} \hat{H}_{l}\left(f-f_{c}\right)\left[U_{l}\left(f-f_{c}\right)+U_{l}^{*}\left(-f-f_{c}\right)\right] \\
& =\frac{1}{2}\left[\hat{H}_{l}\left(f-f_{c}\right) U_{l}\left(f-f_{c}\right)+\hat{H}_{l}^{*}\left(-f-f_{c}\right) U_{l}^{*}\left(-f-f_{c}\right)\right] \\
& =\frac{1}{2}\left[H_{l}\left(f-f_{c}\right) U_{l}\left(f-f_{c}\right)+H_{l}^{*}\left(-f-f_{c}\right) U_{l}^{*}\left(-f-f_{c}\right)\right] \\
& =\frac{1}{2}\left[R_{l}\left(f-f_{c}\right)+R_{l}^{*}\left(-f-f_{c}\right)\right] \\
& =\frac{1}{2} \mathscr{F}\left(r_{l}(t) e^{j 2 \pi f_{c} t}+r_{l}^{*}(t) e^{-j 2 \pi f_{c} t}\right) \\
& =\mathscr{F}\left(\operatorname{Re}\left(r_{l}(t) e^{j 2 \pi f_{c} t}\right)\right),
\end{aligned}
$$

where

$$
\begin{aligned}
H(f) & =\hat{H}_{l}\left(f-f_{c}\right), \\
H(f) & =H^{*}(-f), \\
\hat{H}_{l}\left(f-f_{c}\right) & =\hat{H}_{l}^{*}\left(-f-f_{c}\right) ; \\
\hat{H}_{l}\left(f-f_{c}\right) U_{l}\left(f-f_{c}\right) & =H_{l}\left(f-f_{c}\right) U_{l}\left(f-f_{c}\right), \\
\hat{H}_{l}^{*}\left(-f-f_{c}\right) U_{l}^{*}\left(-f-f_{c}\right) & =H_{l}^{*}\left(-f-f_{c}\right) U_{l}^{*}\left(-f-f_{c}\right) .
\end{aligned}
$$

Note that equation (B5) holds because $\hat{H}_{l}\left(f-f_{c}\right)$ and $\hat{H}_{l}^{*}\left(-f-f_{c}\right)$ have a non-zero frequency response at both positive and negative frequencies, while $U_{l}\left(f-f_{c}\right)$ and $U_{l}^{*}\left(-f-f_{c}\right)$ have a non-zero frequency response only at positive and negative frequencies, respectively.

Finally, equation (B3) demonstrates that the statespace representation (17) of the baseband equivalent "shifted" system can effectively be used to replace the expensive time-domain simulations of the bandpass system.

\section{Appendix C Hamiltonian Matrix of Bandpass Equiv- alent System}

Following the procedure in [17] and assuming that $\hat{\boldsymbol{S}}_{l}(s)$ is the scattering matrix of a baseband equivalent system, 
such system is switching from a non-passive to a passive state (or the other way around) at the frequencies where $\boldsymbol{I}-\hat{\boldsymbol{S}}_{l}^{H}(s) \hat{\boldsymbol{S}}_{l}(s)=0$. To identify these frequencies, with input $\left|\boldsymbol{u}_{l}\right| \neq 0$, we write

$$
\begin{aligned}
\boldsymbol{w}_{l} & =\left(\boldsymbol{I}-\hat{\boldsymbol{S}}_{l}^{H}(s) \hat{\boldsymbol{S}}_{l}(s)\right) \boldsymbol{u}_{l}=0, \\
\boldsymbol{y}_{l 1} & =\hat{\boldsymbol{S}}_{l}(s) \boldsymbol{u}_{l} \\
\boldsymbol{y}_{l 2} & =\hat{\boldsymbol{S}}_{l}^{H}(s) \boldsymbol{y}_{l 1}, \\
\boldsymbol{w}_{l} & =\boldsymbol{u}_{l}-\boldsymbol{y}_{l 2}=0 .
\end{aligned}
$$

Let us assume that $\hat{\boldsymbol{S}}_{l}(s)$ has state-space parameters $\hat{\boldsymbol{A}}_{l}, \hat{\boldsymbol{B}}_{l}, \hat{\boldsymbol{C}}_{l}, \hat{\boldsymbol{D}}_{l}$; while $\hat{\boldsymbol{A}}_{l}^{H}, \hat{\boldsymbol{C}}_{l}^{H}, \hat{\boldsymbol{B}}_{l}^{H}, \hat{\boldsymbol{D}}_{l}^{H}$ are the state-space parameters of $\hat{\boldsymbol{S}}_{l}^{H}(s)$. Then, equations (C2) and (C3) can be written in the form

$$
\begin{aligned}
j \omega \boldsymbol{x}_{l 1} & =\hat{\boldsymbol{A}}_{l} \boldsymbol{x}_{l 1}+\hat{\boldsymbol{B}}_{l} \boldsymbol{u}_{l}, \\
\boldsymbol{y}_{l 1} & =\hat{\boldsymbol{C}}_{l} \boldsymbol{x}_{l 1}+\hat{\boldsymbol{D}}_{l} \boldsymbol{u}_{l} ; \\
-j \omega \boldsymbol{x}_{l 2} & =\hat{\boldsymbol{A}}_{l}^{H} \boldsymbol{x}_{l 2}+\hat{\boldsymbol{C}}_{l}^{H} \boldsymbol{y}_{l 1}, \\
\boldsymbol{y}_{l 2} & =\hat{\boldsymbol{B}}_{l}^{H} \boldsymbol{x}_{l 2}+\hat{\boldsymbol{D}}_{l}^{H} \boldsymbol{y}_{l 1} ;
\end{aligned}
$$

which can be simplified as

$$
\begin{gathered}
j \omega\left[\begin{array}{l}
\boldsymbol{x}_{l 1} \\
\boldsymbol{x}_{l 2}
\end{array}\right]=\left[\begin{array}{cc}
\hat{\boldsymbol{A}}_{l} & 0 \\
-\hat{\boldsymbol{C}}_{l}^{H} \hat{\boldsymbol{C}}_{l} & -\hat{\boldsymbol{A}}_{l}^{H}
\end{array}\right]\left[\begin{array}{l}
\boldsymbol{x}_{l 1} \\
\boldsymbol{x}_{l 2}
\end{array}\right]+\left[\begin{array}{c}
\hat{\boldsymbol{B}}_{l} \\
-\hat{\boldsymbol{C}}_{l}^{H} \hat{\boldsymbol{D}}_{l}
\end{array}\right] \boldsymbol{u}_{(\mathrm{C} 9)} \\
{\left[\begin{array}{ll}
\hat{\boldsymbol{D}}_{l}^{H} \hat{\boldsymbol{C}}_{l} & \hat{\boldsymbol{B}}_{l}^{H}
\end{array}\right]\left[\begin{array}{l}
\boldsymbol{x}_{l 1} \\
\boldsymbol{x}_{l 2}
\end{array}\right]=\left(\boldsymbol{I}-\hat{\boldsymbol{D}}_{l}^{H} \hat{\boldsymbol{D}}_{l}\right) \boldsymbol{u}_{l}}
\end{gathered}
$$

Combining (C9) and (C10) leads to

$$
\begin{aligned}
& j \omega\left[\begin{array}{l}
\boldsymbol{x}_{l 1} \\
\boldsymbol{x}_{l 2}
\end{array}\right]=
\end{aligned}
$$

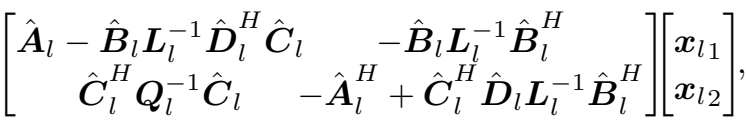

where

$$
\begin{aligned}
\hat{\boldsymbol{L}}_{l} & =\hat{\boldsymbol{D}}_{l}^{H} \hat{\boldsymbol{D}}_{l}-\boldsymbol{I}, \\
\hat{\boldsymbol{Q}}_{l} & =\hat{\boldsymbol{D}}_{l} \hat{\boldsymbol{D}}_{l}^{H}-\boldsymbol{I} .
\end{aligned}
$$

Thus, the Hamiltonian matrix of baseband equivalent systems is

$$
\hat{\boldsymbol{M}}_{l}=\left[\begin{array}{cc}
\hat{\boldsymbol{A}}_{l}-\hat{\boldsymbol{B}}_{l} \hat{\boldsymbol{L}}_{l}^{-1} \hat{\boldsymbol{D}}_{l}^{H} \hat{\boldsymbol{C}}_{l} & -\hat{\boldsymbol{B}}_{l} \hat{\boldsymbol{L}}_{l}^{-1} \hat{\boldsymbol{B}}_{l}^{H} \\
\hat{\boldsymbol{C}}_{l}^{H} \hat{\boldsymbol{Q}}_{l}^{-1} \hat{\boldsymbol{C}}_{l} & -\hat{\boldsymbol{A}}_{l}^{H}+\hat{\boldsymbol{C}}_{l}^{H} \hat{\boldsymbol{D}}_{l} \hat{\boldsymbol{L}}_{l}^{-1} \hat{\boldsymbol{B}}_{l}^{H}
\end{array}\right]
$$

and its imaginary eigenvalues $j \omega$ give the angular frequencies $\omega$ where $(\mathrm{C} 1)$ is satisfied and the matrix $\boldsymbol{I}-$ $\hat{\boldsymbol{S}}_{l}^{H}(s) \hat{\boldsymbol{S}}_{l}(s)$ is singular.
Finally, by indicating the eigenvalues of $\hat{M}_{l}$ with the symbol $\hat{\lambda}_{l}^{z}$, the following equation holds

$$
\hat{\lambda}_{l}^{z}\left[\begin{array}{l}
\boldsymbol{x}_{l 1} \\
\boldsymbol{x}_{l 2}
\end{array}\right]=\hat{\boldsymbol{M}}_{l}\left[\begin{array}{l}
\boldsymbol{x}_{l 1} \\
\boldsymbol{x}_{l 2}
\end{array}\right] \text {. }
$$

Now, assuming that exists a matrix $\boldsymbol{M}$ with eigenvalues $\lambda^{z}$ satisfying $\hat{\boldsymbol{M}}_{l}=\boldsymbol{M}-j 2 \pi f_{c} \boldsymbol{I}$, leads to

$$
\lambda^{z}\left[\begin{array}{l}
\boldsymbol{x}_{l 1} \\
\boldsymbol{x}_{l 2}
\end{array}\right]=\boldsymbol{M}\left[\begin{array}{l}
\boldsymbol{x}_{l 1} \\
\boldsymbol{x}_{l 2}
\end{array}\right]=\hat{\lambda}_{l}^{z}\left[\begin{array}{l}
\boldsymbol{x}_{l 1} \\
\boldsymbol{x}_{l 2}
\end{array}\right]+j 2 \pi f_{c}\left[\begin{array}{l}
\boldsymbol{x}_{l 1} \\
\boldsymbol{x}_{l 2}
\end{array}\right],
$$

which indicates that

$$
\hat{\lambda}_{l}^{z}=\lambda^{z}-j 2 \pi f_{c}, \quad \text { for } z=1, \ldots, Z .
$$

\section{References}

[1] A. F. Oskooi, D. Roundy, M. Ibanescu, P. Bermel, J. Joannopoulos, and S. G. Johnson, "Meep: A flexible free-software package for electromagnetic simulations by the FDTD method," Comput. Phys. Commun. 181, 687 $-702(2010)$.

[2] L. M. Zhang, S. F. Yu, M. C. Nowell, D. D. Marcenac, J. E. Carroll, and R. G. S. Plumb, "Dynamic analysis of radiation and side-mode suppression in a secondorder DFB laser using time-domain large-signal traveling wave model," IEEE J. Quantum Electron. 30, 13891395 (1994).

[3] H. Bahrami, H. Sepehrian, C. S. Park, L. A. Rusch, and W. Shi, "Time-domain large-signal modeling of traveling-wave modulators on SOI," J. Lightwave Technol. 34, 2812-2823 (2016).

[4] S. Balac and F. Mahe, "An embedded split-step method for solving the nonlinear schrodinger equation in optics," J. Comput. Phys. 280, 295 - 305 (2015).

[5] M. Fiers, T. V. Vaerenbergh, K. Caluwaerts, D. V. Ginste, B. Schrauwen, J. Dambre, and P. Bienstman, "Time-domain and frequency-domain modeling of nonlinear optical components at the circuit-level using a node-based approach," J. Opt. Soc. Am. B 29, 896-900 (2012).

[6] S. Mingaleev, A. Richter, E. Sokolov, C. Arellano, and I. Koltchanov, "Towards an automated design framework for large-scale photonic integrated circuits," Proc. SPIE 9516, 951602 (2015).

[7] R. Achar and M. S. Nakhla, "Simulation of high-speed interconnects," Proc. IEEE 89, 693-728 (2001).

[8] B. Gustavsen and A. Semlyen, "Rational approximation of frequency domain responses by vector fitting," IEEE Trans. Power Del. 14, 1052-1061 (1999).

[9] G. Antonini, "SPICE equivalent circuits of frequencydomain responses," IEEE Trans. Electromagn. Compat. 45, 502-512 (2003).

[10] A. Chinea, S. Grivet-Talocia, H. Hu, P. Triverio, D. Kaller, C. Siviero, and M. Kindscher, "Signal integrity verification of multichip links using passive channel macromodels," IEEE Trans. Compon. Packag. Manuf. Technol. 1, 920-933 (2011).

[11] D. Spina, F. Ferranti, G. Antonini, T. Dhaene, L. Knockaert, and D. Vande Ginste, "Time-domain 
Green's function-based parametric sensitivity analysis of multiconductor transmission lines," IEEE Trans. Compon. Packag. Manuf. Technol. 2, 1510-1517 (2012).

[12] M. Sahouli and A. Dounavis, "An instrumental-variable QR decomposition vector-fitting method for modeling multiport networks characterized by noisy frequency data," IEEE Microw. Compon. Lett. 26, 645-647 (2016).

[13] S. Grivet-Talocia and B. Gustavsen, "Black-box macromodeling and its EMC applications," IEEE Electromagn. Compat. Mag. 5, 71-78 (2016).

[14] L. Chrostowski and M. Hochberg, Silicon Photonics Design: From Devices to Systems (Cambridge University Press, 2015).

[15] D. Saraswat, R. Achar, and M. S. Nakhla, "A fast algorithm and practical considerations for passive macromodeling of measured/simulated data," IEEE Trans. Adv. Packag. 27, 57-70 (2004).

[16] D. Deschrijver, M. Mrozowski, T. Dhaene, and D. De Zutter, "Macromodeling of multiport systems using a fast implementation of the vector fitting method," IEEE Microw. Compon. Lett. 18, 383-385 (2008).

[17] B. Gustavsen and A. Semlyen, "Fast passivity assessment for $S$-parameter rational models via a half-size test matrix," IEEE Trans. Microw. Theory Techn. 56, 27012708 (2008).

[18] D. Deschrijver, and T. Dhaene, "Fast passivity enforcement of S-parameter macromodels by pole perturbation," IEEE Trans. Microw. Theory Techn. 57, 620-626 (2009).

[19] R. H. Muller, "Definitions and conventions in ellipsometry," Surf. Sci. 16, $14-33$ (1969).

[20] R. Atkinson and P. H. Lissberger, "Sign conventions in magneto-optical calculations and measurements," Appl. Opt. 31, 6076-6081 (1992).

[21] S. Grivet-Talocia and A. Ubolli, "On the generation of large passive macromodels for complex interconnect structures," IEEE Trans. Adv. Packag. 29, 39-54 (2006).

[22] D. G. Schultz and J. L. Melsa, State Functions and Linear Control Systems (McGrawHill, 1967).

[23] J. Butcher, Numerical Methods for Ordinary Differential Equations (John Wiley and Sons, 2003).

[24] M. C. Jeruchim, P. Balaban, and K. S. Shanmugan, Simulation of communication systems: modeling, methodology and techniques (Springer, 2006).

[25] J. B. King and T. J. Brazil, "Time-domain simulation of passband S-parameter networks using complex baseband vector fitting," in Integrated Nonlinear Microwave and Millimetre-wave Circuits Workshop (INMMiC)(2017), pp. 1-4.

[26] D. Youla, L. Castriota, and H. Carlin, "Bounded real scattering matrices and the foundations of linear passive network theory," IRE Transactions on Circuit Theory 6 , 102-124 (1959).

[27] P. Triverio, S. Grivet-Talocia, M. S. Nakhla, F. G. Canavero, and R. Achar, "Stability, causality, and passivity in electrical interconnect models," IEEE Trans. Adv. Packag. 30, 795-808 (2007).

[28] R. W. Newcomb, "On the energy in passive systems," Proc. IEEE 53, 1651-1652 (1965).

[29] R. Nedunuri, "On the definition of passivity," IEEE Transactions on Circuit Theory 19, 72-72 (1972).
[30] M. R. Wohlers, Lumped and Distributed Passive Networks (Academic, New York, 1969).

[31] S. Boyd and L. O. Chua, "On the passivity criterion for LTI N-ports," Int. J. Circ. Theor. App. 10, 323-333 (1982).

[32] B. Gustavsen, "Fast passivity enforcement for Sparameter models by perturbation of residue matrix eigenvalues," IEEE Trans. Adv. Packag. 33, 257-265 (2010).

[33] www.Iucedaphotonics.com.

[34] The Mathworks Inc., Natick, MA, USA.

[35] K. Jinguji and M. Kawachi, "Synthesis of coherent twoport lattice-form optical delay-line circuit," J. Lightwave Technol. 13, 73-82 (1995).

[36] S. Suzuki, Y. Inoue, and T. Kominato, "High-density integrated $1 \times 16$ optical FDM multi/demultiplexer," in IEEE LEOS Meeting (1994), Vol.2, pp. 263-264.

[37] Y. Xing, D. Spina, A. Li, T. Dhaene, and W. Bogaerts, "Stochastic collocation for device-level variability analysis in integrated photonics," Photon. Res. 4, 93-100 (2016). 